\title{
O PROTOCOLO DE PALERMO NO CONTROLE MIGRATÓRIO MUNDIAL
}

\author{
Fernanda Cláudia Araújo da Silva ${ }^{1}$
}

\section{RESUMO}

As migrações sempre existiram no mundo globalizado, principalmente com o intuito de colonização, estabelecendo-se positivamente e com propósitos político-econômicos. No entanto, ensejou em um certo momento, a necessidade de se estabelecer um marco de governança nas migrações, com a consolidação em diversos níveis, inclusive por meio do Protocolo de Palermo. Dessa forma, procura-se realizar um estudo sobre a interferência do Protocolo no controle migratório. A pesquisa é realizada a partir de um estudo que envolve normativos internacionais sobre migração e sobre organismos internacionais e nacionais como protagonistas que auxiliam no fenômeno migratório.

Palavras-chaves: Protocolo de Palermo; Governança; Migração; Fenômeno; Controle.

\section{THE PALERMO PROTOCOL IN THE WORLD MIGRATION CONTROL}

\begin{abstract}
Migrations have always existed in the globalized world, mainly for the purpose of colonization, establishing themselves positively and for political-economic purposes. However, at a certain moment, there was the need to establish a governance framework for migration, with consolidation at various levels, including through the Palermo Protocol. Thus, an attempt is made to conduct a study on the interference of the Protocol in migratory control. The research is based on a study that involves international norms on migration and on international and national organizations as protagonists that assist in the migratory phenomenon.
\end{abstract}

Keywords: Palermo Protocol; Governance; International migration; Phenomenon; Control.

\section{INTRODUÇÃO}

O trânsito de pessoas no mundo tem sido um acontecimento desencadeado desde as grandes descobertas territoriais que ensejaram colonizações e busca por expansão do mercado. Portanto, desde o início, o processo migratório tem sido multicausal que vão além de descobertas, mas também por consequências de sobrevivência ao longo da história.

\footnotetext{
${ }^{1}$ Mestre em Direito. Doutoranda em Direito pela Universidade de Lisboa. Professora do Departamento de Direito Público da Faculdade de Direito da Universidade Federal do Ceará-UFC. Lattes: http://lattes.cnpq.br/5403306873366136. E-mail: f.c.araujo@ hotmail.com
} 
Os dois períodos bélicos que passou o mundo, ensejou, após a segunda guerra mundial uma maior preocupação, principalmente pelo fato de que a reconstrução da economia mundial precisava ocorrer, por isso, a preocupação mundial pelo controle de fatores que movimentavam países e sacrificavam pessoas em busca da sobrevivência.

Não somente fatores econômicos, mas o fluxo migratório também tem sido motivado, além das guerras, por outras razões como desastres ambientais, perseguições políticas, étnicas ou culturais, que justificam a saída de pessoas de seus territórios para outros lugares.

Atualmente diversos relatórios são expedidos por organismos internacionais e nacionais, com o propósito de estabelecer diretrizes, mapeamentos e normativos que alcancem a situação migratória da contemporaneidade, principalmente por causa dos múltiplos fatores que afetam direitos das pessoas.

Atualmente, existem cerca de 272 milhões de migrantes internacionais no mundo todo, o que corresponde a 3,5\% da população global (OIM/ONU), e o país que mais recebe imigrantes dão os Estados Unidos, seguido da Alemanha e depois a Arábia Saudita, enquanto que, os países que mais têm sua população migrando são, em ordem quantitativa, a Índia, China e em terceiro lugar o México. Quanto ao Brasil, estima-se que 30\% dos imigrantes que chegam, estão no Estado de São Paulo. Há também uma boa parcela de brasileiros no mundo, sendo que 1,8 milhões de imigrantes brasileiros estão em Portugal. Na atualidade, principalmente na América Latina, verifica-se a existência do maior fluxo de pessoas até então, vindos da Venezuela que comprometem a organização dos países recebedores.

Esse referencial migratório impõe aos países a adoção seletiva de recebimento do fluxo migratório, a evitar assimetrias econômicas causadas pela migração internacional hodierna, ainda que as razões tenham sido de violação a direitos.

Porém, a identificação desse fluxo migratório tem contado com a participação de importantes pessoas internacionais que, além de identificar essas contradições dentro das relações internacionais, têm oferecido ajuda humanitária, de forma que atuam dentro de uma transdisciplinaridade. Exatamente esses atores sociais são importantes na ajuda do processo 
migratório mundial, por isso, se estabelece um estudo sobre a importância do terceiro setor, como protagonistas no fluxo migratório.

A pesquisa adota com metodologia uma pesquisa descritiva, a partir da leitura de normativos, artigos e tratados, convenções e protocolos que embasam o trabalho. Quanto à estrutura, o trabalho está dividido em três partes, além da introdução e considerações finais. $\mathrm{Na}$ primeira parte realiza-se uma análise sobre o processo migratório mundial, em seguida, faz-se um estudo sobre o marco da governança mundial sobre migrações e, por último uma abordagem sobre o Protocolo de Palermo.

\section{O PROCESSO MIGRATÓRIO MUNDIAL CONTEMPORÂNEO}

A migração entendida como fenômeno demográfico se estabelece mundialmente de várias maneiras e diante diversas situações históricas, de forma que ocorrer com a mudança da unidade geográfico-territorial do indivíduo dentro de seu próprio país, o que se denomina de migração interna, ou para Estado estrangeiro, denominando-a de migração internacional.

A análise do processo migratório pode ser estudada sob vários momentos da história, no entanto, se opta por estabelecer um estudo do processo migratório mundial contemporâneo a partir da segunda grande guerra (1939-1945), coincidindo com a criação da Liga das Nações e, posterior, Organização das Nações Unidas ${ }^{2}$, como organismo de alcance global estabelecido, capaz de discutir questões de relevância mundial e de proteção dos direitos humanos, além de fornecer ajuda humanitária, promover o desenvolvimento sustentável em todos os aspectos e proteção do direito internacional (ONU).

Assim, o referencial da migração na contemporaneidade se estabelece por causa de problemas que ocorrem nos países, a partir da segunda grande guerra, identificados por assimetrias econômicas, que deixam os Estados a mercê das dificuldades econômicas principalmente pela falta de oportunidades, ou, mesmo que existam oportunidades, essas não

\footnotetext{
${ }^{2} \mathrm{O}$ nome foi dado pelo então presidente dos Estados Unidos, Franklin D. Roosevelt, utilizado pela primeira vez em $1^{\circ}$ de janeiro de 1942, representando 26 nações. Com o fim da segunda guerra mundial, o organismo foi muito importante para trazer a paz, o que ensejou uma 'proposta de trabalho global.
} 
são suficientes para atender toda coletividade. Expressam-se no processo migratório outros elementos como fatores políticos, sociais, étnicos, religiosos, ambientais etc.

Acompanham os imigrantes, além da ultrapassagem de fronteira, os valores culturais, linguísticos, ideológicos, informacionais que se instalam em novo território, apesar de todas as dificuldades que cercam o processo de instalação e adaptação de no novo local, de forma que o tempo e a tolerância são os únicos elementos de adaptação do processo migratório. Esse receio cultual e a nova identidade a ser enfrentada pela migração a se evitar enquistamentos ${ }^{3}$ culturais e a formação de guetos migratórios.

Há, dentro desse contexto de mudança geográfica, a definição do próprio conceito de migrante a ser estabelecido dentro da proposta organizacional da ONU, mas também sem ser uma definição fechada, a se referir a toda pessoa que muda de seu local habitual de residência por diferentes motivos e objetivos dando uma maior exatidão ao termo, como, por exemplo, trabalhadores migrantes, refugiados ${ }^{4}$, apátridas etc $^{5}$

Acentuam-se os processos migratórios também no final do século passado enraizado por diversos motivos, representados sinteticamente por Roberto Marinucci e Rosita Milesi:

\begin{abstract}
O final do século XX e o começo do novo milênio estão caracterizados por um clima de desilusão e desconfiança, conseqüência de situações vividas e sofridas durante o século findo. Apesar dos ideais libertários e igualitários do mundo moderno, a humanidade conheceu os crimes hediondos de Auschwitz, de Hiroshima, do campo de concentração de Gulag, das ditaduras militares, da depredação do meio ambiente e do empobrecimento dos povos do sul do mundo. Esta sensação de desilusão foi impulsionada também pela conjuntura política. A queda do muro de Berlin representou um golpe para os que acreditavam na possibilidade de planejar sociedades igualitárias e justas. Sobrou a dura lei do mercado, logo proclamada como única vencedora. (MARINUCCI, MILESI, 2005, p. 4)
\end{abstract}

\footnotetext{
${ }^{3}$ Núcleos populacionais fechados por causa de sua cultura.

${ }^{4}$ Expressão definida em seu sentido jurídico a partir de 1951.

5 "Migration is one of the defining issues of the twenty-first century. It is now an essential, inevitable and potentially beneficial component of the economic and social life of every country and region. The question is no longer whether to have migration, but rather how to manage migration effectively so as to enhance its positive and reduce its negative impacts"Disponível em: 〈www.acnur.org/t3/portugues/documentos/?tx_danpdocumentdirs_pi2\%5Bpointer\%〉. Acesso em janeiro de 2009 .
} 
Há uma predominância exposta que motiva a migração, condicionando-a ao gerenciamento econômico, mesmo que a trate como um fenômeno permanente da sociedade globalizada, mas, com o intuito de involucrar o Estados de acolhida e a figura do imigrante e que precisam ser esses dois lados, não podendo ficar a temática sem referência, e sim, a ser gestionada, com o envolvimento da sociedade e dos organismos existentes na atualidade.

As migrações são muitas, mas algumas apresentam um referencial que pontua preocupações no cenário mundial, como o Sudão do Sul, cenário de uma guerra civil durante quase cinco anos, é caracterizado pelas atrocidades de caráter étnico e obrigou cerca de 4,2 milhões de pessoas, quase um terço da população, a fugir para Uganda, Etiópia e Sudão, considerada uma das piores crises humanitárias.

A Europa tem sido o berço recebedor de imigrantes, num fluxo maciço e continuado, o que ensejou políticas mais severas no recebimento de migrantes e até mesmo no restabelecimento de fronteiras, principalmente pela existência de uma migração familiar:

El proceso de europeización de la política migratoria ha incidido también en las políticas de migración familiar. Debates, concepciones y regulaciones en torno a la migración por motivos familiares que toman cuerpo en un contexto nacional específico, son replicados (y reificados) en otros países de la región. Los ejemplos más gráficos de lo que aquí se señala son los contratos de integración y los exámenes de lengua en los países de origen como condición para acceder a un visado por reunificación o formación familiar. En general, los motivos esgrimidos para reforzar el control sobre la migración por motivos familiares giran en torno a tres ejes: 1) la creación de unas bases más sólidas para la integración, 2) la defensa del Estado de bienestar y 3) la reducción de los matrimonios forzados y arreglados por parte de las familias de las jóvenes inmigrantes o hijas de inmigrantes, en su mayoría musulmanes. (ARAUJO, PEDONE, 2014, p.3)

Outro grande processo migratório de repercussão na contemporaneidade é a migração venezuelana, considerado o maior fluxo de pessoas na América Latina, desde 2015. Tanto que o Acnur acredita que esse êxodo de venezuelanos tem razões meramente econômicas e expõe:

Dado o agravamento da situação política, econômica, humanitária e de direitos humanos na Venezuela, que já deslocou globalmente mais de 3,6 milhões de 
pessoas, a Agência da ONU para Refugiados (ACNUR) considera que a maioria dos que fogem do país precisa de proteção internacional para refugiados. [...] o ACNUR reitera seu apelo aos Estados para que permitam o acesso dos venezuelanos a seu território e forneçam proteção e tratamento adequado, destacando a necessidade crítica de segurança das pessoas forçadas a fugir por suas vidas e por liberdade. (ONU, 2019)

Outro processo migratório significativo que tem ocorrido desde 2018, é o de Honduras, em que milhares de hondurenhos têm saído do país, escapando da violência e da pobreza, e, via de regra, o destino final são os Estados Unidos.

A movimentação hodierna decorre, portanto, dos problemas econômicos existentes nos países de origem, mesmo que em decorrência de outros motivos como guerras, problemas religiosos etc. Mas, com fluxos massivos de migração existente, e como afirma Cristina Valderrama:

\begin{abstract}
Ahora bien, una política de inmigración en sentido amplio excede con mucho a un marco jurídico, pues éste se limita a regular las condiciones específicas de entrada, estancia y salida de los extranjeros en territorio nacional. Una política de inmigración debe tener en cuenta no sólo el control de flujos y estancias; debe atender a la forma en que los extranjeros desarrollan su vida en el país de destino y a la convivencia con los nacionales, y debe estar atenta a las causas que producen indeseados y masivos movimientos migratorios internacionales. En el primer caso se habla de una política de integración, encaminada a facilitar las condiciones de vida de los inmigrantes y su convivencia con los nacionales, mientras que en el segundo estaríamos haciendo referencia a una política de cooperación internacional tendente a mitigar, en los lugares de origen, las causas de la emigración forzosa. (VALDERRAMA, 2001, p. 238)
\end{abstract}

Portanto, essa movimentação carece de uma governança para gerenciamento migratório nos países, não por meio de um único organismo, mas de um trabalho em rede, como a Declaração de Nova York, a qual estabelece um Comprehensive Refugee Response framework acerca da migração.

\title{
2 GOVERNANÇA NAS MIGRAÇÕES
}


Ante à existência de uma movimentação de pessoas no mundo é necessário estabelecer um marco referencial de governança, ao mesmo tempo em que se protegem pessoas migrantes e Estados que participam dessa movimentação.

O marco de governança se estabelece desde as definições de institutos jurídicos passando pela existência de uma organização institucional como a Organização Internacional de Migração - OIM, com o intuito de melhorar as regras de governança migratória e interferir na regulação de Estados-membros da OIM, com delineamento dos princípios da adesão e respeito às normas internacionais e ao bem-estar socioeconômico dos imigrantes.

No entanto, não existe uma estrutura uníssona de governança global de gerenciamento da migração mundial, mas, diversos documentos que tratam de exílio, migração irregular, migração por trabalho etc, como se existissem sistemas estruturais independentes, ora emitidos pelo ACNUR - Alto Comissariado das Nações Unidas para os Refugiados, ora pela OIM - Organização Internacional para as Migrações (BETTS, 2010). Porém, percebe-se a existência de diferentes assuntos abordados que se estabelecem em cada caso de emissão de normas sobre migrações mundiais.

A proposta de um marco de governança sobre circulação de pessoas enseja uma adoção de estruturas multilaterais a estabelecer uma maior abrangência e cooperação entre os pais, principalmente quando se estabelece alcance integrativo local ou regional, o que se teria como exemplo, a União Europeia e o Mercosul, que tratam em seus documentos regras acerca de mobilidade e migração ${ }^{6}$.

A análise da governança migratória segue a difusão de esquemas globais, ou seja, de cima para baixo, top-down a alcançar setores gerais, e os regionais a estabelecerem diretrizes mais específicas.

A governança regional traz gerenciamentos ao alcance local e a estabelecer maior integração entre os Estados envolvidos (AWUMBILA et. al, 2014), o que justificaria os interesses da região e dos Estados dentro de referências setoriais, diferentemente da

\footnotetext{
${ }^{6}$ No entanto, cabe mencionar aqui que a proposta migratória a envolver a Venezuela não pode ser identificada como uma migração regional pois o país Bolívar não faz parte do bloco econômico do Mercosul.
} 
governança global, pois se estabelece uma diretriz a partir das necessidades encontradas, trazendo um gerenciamento mais eficaz, pois seria de baixo para cima, bottom-up.

A construção de uma governança migratória regional analisa regras, normativos e práticas sociais que ajudariam a melhorar e coordenar os envolvidos na política migratória e explicar melhor a necessidade migratória e suas alocações (BRAZ, 2018).

Hall e Bevir (2011) estabelecem três perspectivas diferentes: a) um foco econômico, para facilitar a mobilidade como fator de produção; b) uma ênfase baseada nos direitos civis e sociais dos migrantes; e c) um foco baseado na segurança e controle migratório. O propósito é entender as necessidades locais e estabelecer políticas públicas mais efetivas, e trazer uma perspectiva intergovernamental para proteção dos interesses locais e econômicos da região.

Diferentemente da política global migratória, os direcionamentos alcançam elementos principiológicos e genéricos, mas sem uma cooperação regional a atender a peculiaridades locais, e mesmo que se respeite o "processo pelo qual uma inovação é comunicada através de certos canais ao longo do tempo entre os membros de um sistema social" (ROGERS, 1983, p. 5), ou seja, dentro de uma proximidade geográfica, semelhanças políticas, institucionais ou ideológicas são necessárias para a adoção de políticas públicas migratórias. Portanto, as políticas migratórias bottom-up são mais eficientes que as políticas migratórias top-down.

A atuação de organismos internacionais, inclusive outros atores considerados pertencentes ao terceiro setor que ajudam na migração mundial, tomam decisões de acordo com suas peculiaridades. Como exemplo, tomado tem-se a migração venezuelana que entre os sujeitos que migraram existem indígenas, com costumes e linguagem diferentes, tanto que o abrigo construído para eles (Abrigo da Pintolândia) foi diferente dos demais abrigos, respeitando-se as tradições, a cultura e a forma de vivência, como a dormida, pois só colocaram redários e não camas. Diferenças são importantes na manutenção da proteção dos direitos dos imigrantes, o que valoriza uma conduta regionalizada.

A ótica da observação que ora se perfaz, refere-se a uma abordagem regionalista das necessidades dos imigrantes, e não uma separação entre governança europeia-ocidental ou 
outra forma. O entendimento de alguns autores que os modelos não podem ser comparados e sim integrados entre si (BÖRZEL, RISSE, 2016).

No entanto, isso não quer dizer que divergências não possam ocorrer quando confrontados os modelos de governança internacional e regional, principalmente pelo fato de que os países que compõem uma regionalidade podem trazer elementos específicos a serem disciplinados, resultando uma integração muito maior e de melhor solução (GEDDES, ARCARAZO, 2014). A governança também pode ser considerada sub-regional, por atender parte dos Estados de uma região.

Já governança migratória doméstica também traz direcionamentos ao fluxo migratório, respeitando-se a ordem interna de cada Estado. Graficamente os níveis de governança são assim estabelecidos por vários autores:

Figura 1: Representatividade dos níveis de governança

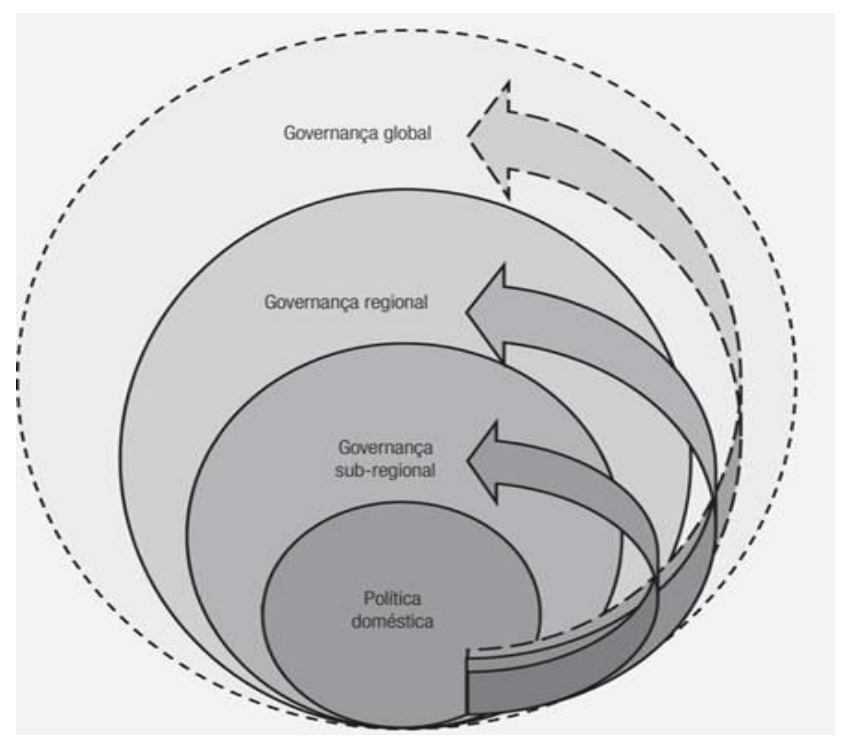

Fonte: Porto de Oliveira (2017); Geddes e Arcarazo (2014); Margheritis (2012); Brumat (2016); Perrotta (2016).

Assim, percebe-se a ocorrência de adequação das políticas de governança proposta pelos autores a estabelecer uma participação de forma de política, em que uma pertence a outra e que inversamente as diretrizes normativas também estão presentes a considerar as 
governanças internacional, regional sub-regional e doméstica, pois a proteção migratória deve ser estabelecida em todos os níveis.

O ACNUR- Alto Comissariado das Nações Unidas para os Refugiados e a Organização Internacional para Migração - OIM, mesmo com direcionamentos constantes e efetivos em todos os continentes, têm respeitado convenções e declarações regionais adotados nos últimos anos, o que significa que as entidades compreendem a atualidade a atualidade da situação da movimentação de pessoas e que muitas vezes decisões internas de países ou regionais são benéficas, devem ser respeitadas, reconhecendo também que os direcionamentos regionais, sub-regionais e internos balizam ações de proteção aos Direitos Humanos.

\section{O PROTOCOLO DE PALERMO NO CONTROLE MIGRATÓRIO MUNDIAL}

Tem ocorrido um viés contrário às avessas durante concomitante ao fluxo migratório, ocorrendo o tráfico de pessoas. Assim, os organismos internacionais também se preocuparam com esse tipo de migração a partir do Protocolo de Palermo, documento ao qual o Brasil é signatário e ratificado internamente pelo Decreto $\mathrm{n}^{\circ} 5017 / 2004$. O protocolo foi aprovado pela Resolução Geral da ONU n ${ }^{\circ}$ 55/25, em 15 de novembro de 2000, mas somente entrou em vigor em 29 de setembro de 2003.

O documento traz uma peculiaridade em ser considerado o primeiro instrumento global numa definição consensual sofre o tráfico de pessoas no mundo ${ }^{7}$, facilitando a convergência de abordagens sobre a temática, inclusive como mecanismo eficaz contra o tráfico de pessoas, contrabando de imigrantes, proteções à exploração de imigrantes vítimas de vantagens financeiras, com respeito aos direitos humanos. No entanto, Ela Castilho elenca documentos anteriores de proteção ao tráfico de pessoas:

\footnotetext{
${ }^{7}$ No Brasil, a Lei $n^{\circ} 13.334 / 2016$ dispõe sobre prevenção e repressão ao tráfico interno e internacional de pessoas e sobre medidas de atenção às vítimas e altera vários dispositivos doo Código de Processo Penal e Código Penal, além de outras legislações, estabelecendo medidas de enfrentamento ao tráfico de pessos, criação de tipos penais, ao estabelecer no Art. $1^{\circ}$ e seu parágrafo único que "Esta Lei dispõe sobre o tráfico de pessoas cometido no território nacional contra vítima brasileira ou estrangeira e no exterior contra vítima brasileira. Parágrafo único. O enfrentamento ao tráfico de pessoas compreende a prevenção e a repressão desse delito, bem como a atenção às suas vítimas"
} 
À preocupação inicial com o tráfico de negros da África, para exploração laboral, agregou-se a do tráfico de mulheres brancas, para prostituição. Em 1904, é firmado em Paris o Acordo para a Repressão do Tráfico de Mulheres Brancas, no ano seguinte convolado em Convenção. Durante as três décadas seguintes foram assinados: a Convenção Internacional para a Repressão do Tráfico de Mulheres Brancas (Paris, 1910), a Convenção Internacional para a Repressão do Tráfico de Mulheres e Crianças (Genebra, 1921), a Convenção Internacional para a Repressão do Tráfico de Mulheres Maiores (Genebra, 1933), o Protocolo de Emenda à Convenção Internacional para a Repressão do Tráfico de Mulheres e Crianças e à Convenção Internacional para a Repressão do Tráfico de Mulheres Maiores (1947), e, por último, a Convenção e Protocolo Final para a Repressão do Tráfico de Pessoas e do Lenocínio (Lake Success, 1949). (CASTILHO, s/d, p.1-2)

Porém, como entende Ela Castilho, essas convenções foram divididas em duas fases, sendo antes e depois da Convenção de 1949, estabelecendo o contexto da Liga das Nações e no âmbito da ONU, com expressa anulação e substituição das normas anteriores.

No entanto, a política proposta pelo Protocolo de Palermo acaba por englobar as matérias previstas anteriormente, pois visa combater o crime organizado transnacional complementada por três outros protocolos correlatos ao crime organizado como o Protocolo Relativo à Prevenção, Repressão e Punição do Tráfico de Pessoas, em Especial Mulheres e Crianças; o Protocolo Relativo ao Combate ao Tráfico de Migrantes por Via Terrestre, Marítima e Aérea; e o Protocolo contra a fabricação e o tráfico ilícito de armas de fogo, suas peças e componentes e munições.

Os dois primeiros protocolos relacionam-se com a gravidade do problema do crime organizado transnacional envolvendo pessoas e promover uma cooperação internacional para o enfrentamento com a adoção de instrumentos que se comprometem em adotar medidas contra o crime organizado transnacional, tipificando a conduta na legislação nacional, estabelecendo que os governos adotem medidas para facilitar processos de extradição, assistência legal mútua e cooperação policial, inclusive na promoção de atividades de capacitação e aprimoramento de policiais e servidores públicos oferecer respostas eficazes contra o crime organizado. Assim, o protocolo define tráfico de pessoas no Art. $3^{\circ}$, alínea 'a:

o recrutamento, o transporte, a transferência, o alojamento ou o acolhimento de pessoas, recorrendo à ameaça ou ao uso da força ou a outras formas de coação, ao rapto, à fraude, ao engano, ao abuso de autoridade ou de situação de 
vulnerabilidade ou à entrega ou aceitação de pagamentos ou benefícios para obter o consentimento de uma pessoa que tem autoridade sobre outra, para fins de exploração. A exploração deverá incluir, pelo menos, a exploração da prostituição de outrem ou outras formas de exploração sexual, o trabalho ou serviços forçados, a escravatura ou práticas similares à escravatura, a servidão ou a extração de órgãos.

O Estado brasileiro ratificou o protocolo e editou norma interna a reprimir o tráfico de pessoas pelo ordenamento jurídico nacional em sua forma de exploração sexual, por meio de crimes, tanto que, por meio da Lei $\mathrm{n}^{\circ} 13.344 / 2016$, passam a ser punidas outras formas de exploração como remoção de órgãos, trabalho escravo, servidão e adoção ilegal, atendendo ao disposto no pacto internacional.

Ela Castilho apresenta as fases do controle internacional do tráfico de pessoas e pondera o Protocolo de Palermo como sendo a terceira fase e assevera de forma lúcida:

\begin{abstract}
Este Protocolo inicia a terceira fase do controle jurídico internacional em matéria de tráfico e de prostituição. Considerando a fase anterior quatro aspectos se destacam. Os dois primeiros dizem respeito às pessoas objeto de proteção. As vítimas que eram, inicialmente, só as mulheres brancas, depois mulheres e crianças, são agora os seres humanos, mantida a preocupação especial com mulheres e crianças. Antes as vítimas ficavam numa situação ambígua, como se fossem criminosas. O Protocolo busca garantir que sejam tratadas como pessoas que sofreram graves abusos, os Estados membros devem criar serviços de assistência e mecanismos de denúncia. O terceiro é concernente à finalidade do tráfico. Nas Convenções até 1949 a preocupação era coibir o tráfico para fins de prostituição. O Protocolo acolhe a preocupação da Convenção Interamericana sobre o Tráfico Internacional de Menores para combater o tráfico de pessoas com propósitos ilícitos, neles compreendidos, entre outros, a prostituição, a exploração sexual (não mais restrita à prostituição) e a servidão. O Protocolo emprega a cláusula para fins de exploração, o que engloba qualquer forma de exploração da pessoa humana, seja ela sexual, do trabalho ou a remoção de órgãos. A enumeração é apenas ilustrativa. (CASTILHO, s/d., p. 5)
\end{abstract}

Há uma demonstração não taxativa de hipóteses que caracterizam o crime transacional sobre tráfico de pessoas e que se luta no combate a punição de criminosos que se utilizam do fluxo migratório para praticar o crime de tráfico de pessoas, por isso impõe aos Estados signatários a regulamentação interna da matéria.

De qualquer forma, são estabelecidas políticas de combate a este tipo de crime, considerado um dos tipos criminosos mais lucrativos por conta de suas práticas. 
A normatização interna de cada país deve atender os requisitos determinantes da lei o que pode ser identificado na lei interna com a punição de agentes, principalmente para elencar medidas a serem tomadas com relação à proteção das vítimas em proteção à privacidade, a intimidade e a confidencialidade dos procedimentos judiciais, direito de acesso à informação sobre os processos judiciais e administrativos e assistência para se manifestar durante o trâmite destes; assistência para a recuperação física, psicológica e social das vítimas, incluindo alojamento, aconselhamento e informação, assistência médica, psicológica e material, oportunidade de emprego, educação e formação; cuidados especiais com as necessidades específicas das vítimas, especialmente crianças; garantia da segurança enquanto estiverem no seu território; possibilidade de obter indenização judicial; possibilidade de permanecer de forma temporária ou permanente no seu território, tomando em conta fatores humanitários e pessoais; facilitação do repatriamento, possibilidade de que fiquem, com apoio de entidades sociais. (TRINDADE, 2013).

\section{CONSIDERAÇÕES FINAIS}

Assim, o trânsito de pessoas no mundo tem sido um acontecimento desencadeado desde longas datas, mas que se tornou um referencial a partir da Liga das Nações/ONU principalmente na proposta de estabelecer uma proteção jurídica internacional.

Essa proteção tem sido tratada por meio de governanças migratórias a partir de organismos internacionais em diversos níveis dentro desde uma governança internacional, e outras formas de governança como a regional, sub-regional e interna. Esses mecanismos de gerenciamento dos fluxos migratórios atendem a concessão de dar melhores condições e proteção ao imigrante.

Estabeleceu-se também, um estudo sobre o Protocolo de Palermo e seus efeitos no que se referem a tutela migratória em prevenir o tráfico de pessoas durante o fluxo migratório.

O Protocolo de Palermo foi ratificado pelo brasileiro, o que ensejou internamente na edição da Lei $n^{\circ} 13.344 / 2016$, que regulamentou o combate ao tráfico de pessoas em todas as suas formas, além de estabelecer a proteção de vítimas no cenário do tráfico de pessoas. 
A relação entre governança e o Protocolo de Palermo se estabelece pelo fato do documento internacional priorizar diretrizes protetivas de forma macro, enquanto, a partir do documento internacional podem seguir outros instrumentos normativos de âmbito regional, sub-regional e local, ou interno, como ocorre com a legislação existe no Brasil para combater o tráfico de pessoas no Brasil. Além disso, a tendência moderna do trânsito de pessoas do mundo tende a se desvirtuar e ocorrer crimes como esses protegidos pelo Protocolo de Palermo.

\section{REFERÊNCIAS}

ARAUJO, Gil Araujo, S. Gil, PEDONE, C., 2014. Introducción. Familias migrantes y Estados: vínculos entre Europa y América Latina. In: En Papeles del CEIC, vol. 2014/2, CEIC - Centro de Estudios sobre la Identidad Colectiva, Universidad del País Vasco, http://dx.doi.org/10.1387/pceic.13020. Disponível em: https://ri.conicet.gov.ar/bitstream/handle/11336/42482/CONICET_Digital_Nro.106b65be2109-4f7e-a90b-24105f10884b_A.pdf?sequence=2. Acesso em: 29 de abril de 2020.

AWUMBILA, Mariama et al. Across artificial borders: an assesment of labour migration in Ecowas region. Ghana: IOM.ACP, 2014.

BETTS, Alexander. Migration governance: alternative futures. Le Grand-Saconnex: OIM, 2010. (Background Papers WMR 2010). Available at: $<$ Available at:http://publications.iom.int/system/files/pdf/wmr2010_migration_governance_alternative_fu tures.pdf >. Acceso em: 30 de abril de 2020.

BÖRZEL, Tanja A.; RISSE, Thomas. Introduction: framework of the handbook and conceptual clarifications. In: BÖRZEL, Tanja A.; RISSE, Thomas (Org.). The Oxford handbook of comparative regionalism. Oxford: Oxford University Press, 2016. p. 3-15.

BRAZ, Adriana Montenegro. A governança migratória na américa do sul: a difusão de baixo para cima (bottom-up) do acordo de residência do mercosul. Rev. Adm. Pública, Rio de Janeiro, v. 52, n. 2, p. 303-320, Apr.2018. Available from $<$ http://www.scielo.br/scielo.php?script=sci_arttext\&pid=S0034-

76122018000200303\&lng=en\&nrm=iso>. access on 30 Apr. 2020.

BRUMAT, Leiza Maricel. Políticas migratorias y libertad de circulación en el Mercosur (1991-2012). 2016. Thesis (Doctor Degree in Political Science) - Programa de Doctorado en Ciencias Sociales, Facultad Latinoamericana de Ciencias Sociales, Sede Académica Argentina, Buenos Aires, Available at: <Available 
at: http://repositorio.flacsoandes.edu.ec/handle/10469/10634\#.Wob953xumM8 >. $\quad$ Accesed on: 30 abr. 2020.

CASTILHO, Ela Wiecko V. de. Tráfico de pessoas: da Convenção de Genebra ao Protocolo de Palermo. Disponível em: http://pfdc.pgr.mpf.mp.br/atuacao-e-conteudos-deapoio/publicacoes/trafico-de-pessoas/artigo_trafico_de_pessoas.pdf. Acesso em: 30 de abril de 2020.

GEDDES, Andrew; ARCARAZO, Diego Acosta. Transnational diffusion or different models? Regional approaches to migration governance In the European Union and Mercosur. European Journal of Migration and Law, v. 16, n. 1, p. 19-44, 2014.

HALL, Ian; BEVIR, Mark. Global governance. In: BEVIR, Mark (Org.). The Sage handbook of governance. London: Sage Publications, 2011. Available at: <Available at: http://sk.sagepub.com/reference/hdbk_governance >. Accessed on: 30 BRIL 2020.

LAVENEX, Sandra et al. Regional migration governance. In: BÖRZEL, Tanja A.; RISSE, Thomas (Org.). The Oxford handbook of comparative regionalism. Oxford: Oxford University Press, 2016. p. 457-485.

MARGHERITIS, Ana. Piecemeal regional integration in the post-neoliberal era: negotiating migration policies within Mercosur. Review of International Political Economy, v. 20, n. 3, p. 541-575, 2012.

MARINUCCI, Roberto, MILESI, Rosita. Migrações Internacionais Contemporâneas. In: Instituto Migrações e Direitos Humanos. Disponível em: https://www.migrante.org.br/refugiados-e-refugiadas/migracoes-internacionaiscontemporaneas/. Junho/2005. Acesso em: 29 de abril de 2020.

ONUBRASIL. História da organização. Disponível em: https://nacoesunidas.org/conheca/historia/. Acesso em: 29 de abril de 2020.

ONU. Maioria das pessoas que foge da Venezuela necessita de proteção internacional para refugiados. Disponível em: https://nacoesunidas.org/maioria-das-pessoas-que-fogedavenezuela-necessita-de-protecao-internacional-para-refugiados/. Acesso em: 22 de maio de 2019.

PERROTTA, Daniela. Mercosur, regulatory regionalism and contesting projects of higher education governance. In: ROBERTSON, Susan et al. (Org.). Global regionalisms and higher education projects, processes, politics. London: Edward Elgar Publishing, 2016. p. 228-252. Available at: <Available at: http://www.elgaronline.com/view/9781784712341.00020.xml >. Accessed on: 30 abr. 2020 .

PORTO DE OLIVEIRA, Osmany. International policy diffusion and participatory budgeting: ambassadors of participation, international institutions and transnational networks. Switzerland: Springer, 2017. 
ROGERS, Everett M. Diffusion of innovations. 3. ed. New York: Free Press, 1983.

TRINDADE, A. A. Cançado. Tratado de Direito Internacional dos Direitos Humanos. Vol. I. 2a ed. rev. e atual. Porto Alegre: SAFE, 2003.

VALDERRAMA, Cristina Blanco Fernández de. La Integración de los inmigrantes. Fundamentos para abordar uma política global de Intervención In: Migraciones. Espanha, 2001 . 